\title{
Effects of a Hydrogel Patch on Denture-Related Traumatic Ulcers; an Exploratory Study
}

\author{
Anca Jivanescu, DMD, PhD, ${ }^{1}$ Wenche Sylling Borgnakke, DDS, MPH, PhD, ${ }^{2}$ Luciana Goguta, DMD, PhD, ${ }^{1}$ \\ Raluca Erimescu, DMD, PhD, ${ }^{1}$ Lior Shapira, DMD, PhD, ${ }^{3} \&$ Emanuel Bratu, $\mathrm{DMD}, \mathrm{PhD}^{4}$ \\ ${ }^{1}$ Assistant Professor, Department of Prosthodontics, Faculty of Dentistry, University of Medicine and Pharmacy "Victor Babes," Timisoara, Romania \\ ${ }^{2}$ Adjunct Clinical Assistant Professor, Department of Periodontics and Oral Medicine, School of Dentistry, University of Michigan, Ann Arbor, MI \\ ${ }^{3}$ Professor and Chair, Department of Periodontology, Hebrew University-Hadassah Faculty of Dental Medicine, Jerusalem, Israel \\ 4Professor, Department of Implant Supported Restorations, Faculty of Dentistry, University of Medicine and Pharmacy "Victor Babes," Timisoara, \\ Romania
}

\begin{abstract}
Keywords
Dental prosthesis; inflammation; mouth, edentulous; mouth mucosa; prosthodontics; quality of life.
\end{abstract}

\section{Correspondence \\ Anca Jivanescu, Department of \\ Prosthodontics, Faculty of Dentistry, University of Medicine and Pharmacy "Victor Babes" Timisoara, P-ta Eftimie Murgu Nr. 2, Timisoara, Romania. \\ E-mail: ajivanescu@yahoo.com}

The authors deny any conflicts of interest.

Accepted January 11, 2014

doi: 10.1111/jopr.12186

\begin{abstract}
Purpose: The aim of this exploratory study was to evaluate the effects of hydrogel patch wound dressing on healing time and pain level of denture-related lesions of the oral mucosa in edentulous individuals.

Materials and Methods: Twenty-three adults with newly fabricated complete sets of dentures who subsequently developed at least two ulcerative lesions related to their complete dentures were included in the study. For each participant, the smaller lesion (control lesion) was allocated to usual care, that is, adjustment of the denture's margins, whereas the larger lesion (test lesion) was assigned to receive usual care plus application of a hydrogel patch. In the latter, a patch was applied directly on the affected area three times within the first 24 hours, followed by application of three additional patches, namely one during each of the following 3 days. Participants were monitored until complete healing of all ulcers. The primary outcome measures were changes since baseline in each lesion's greatest dimension at days 1 and 7 , as well as improvement in ulcer-related pain experienced.

Results: Participants were on average about 70 years old, about half were women, and just over $40 \%$ had type 2 diabetes. Lesions treated with the hydrogel patch extended between 4.3 and $10.2 \mathrm{~mm}$ (mean $7.1 \mathrm{~mm}$ ) in their greatest dimension, and the smaller lesions receiving usual care were initially $4 \mathrm{~mm}$ on average, ranging from 2.0 to $7.0 \mathrm{~mm}$. The hydrogel patch lesions attained $25 \%$ to $75 \%$ reductions in their greatest lesion extent from baseline to days 1 and 7, respectively, compared to $10 \%$ and just over 50\% reduction in the lesions that received usual care. Healing rates were similar in patients with and without diabetes. The participants reported significant improvement in pain level 1 day following treatment initiation for 30\% of the control lesions, compared to $65 \%$ of the lesions treated with the hydrogel patch.

Conclusions: The results of this exploratory study suggest that application of hydrogel patches may represent a novel, effective treatment for accelerating the healing process and pain reduction in mucosal lesions associated with complete dentures also in people with type 2 diabetes; however, larger studies need to confirm these findings.
\end{abstract}

Wearing complete dentures improves the quality of life and restores masticatory function in edentulous patients; however, removable dentures may injure oral tissues, and their use is associated with a high frequency of oral mucosal lesion. ${ }^{1}$ Accordingly, the prevalence of oral mucosal lesions is usually reported to be higher in denture wearers than in nonwearers. ${ }^{2}$

Multiple factors can cause mucosal irritation and, eventually, tissue ulcerations. These factors may be provider- or denturerelated, such as imperfect impressions, poor adaptation to sup- porting oral tissue, poor flange extension, occlusion disharmonies, and unpolished surfaces. They can also be related to the denture wearer, such as wearing time and masticatory and dietary habits, as well as poor denture hygiene measures. ${ }^{3}$ Systemic risk factors are age, smoking habits, and comorbidities, such as diabetes mellitus. ${ }^{4}$

Mucosal injuries following the insertion of complete dentures may occur in all supporting tissues in contact with the base and borders of the dentures, as well as tissues coming 


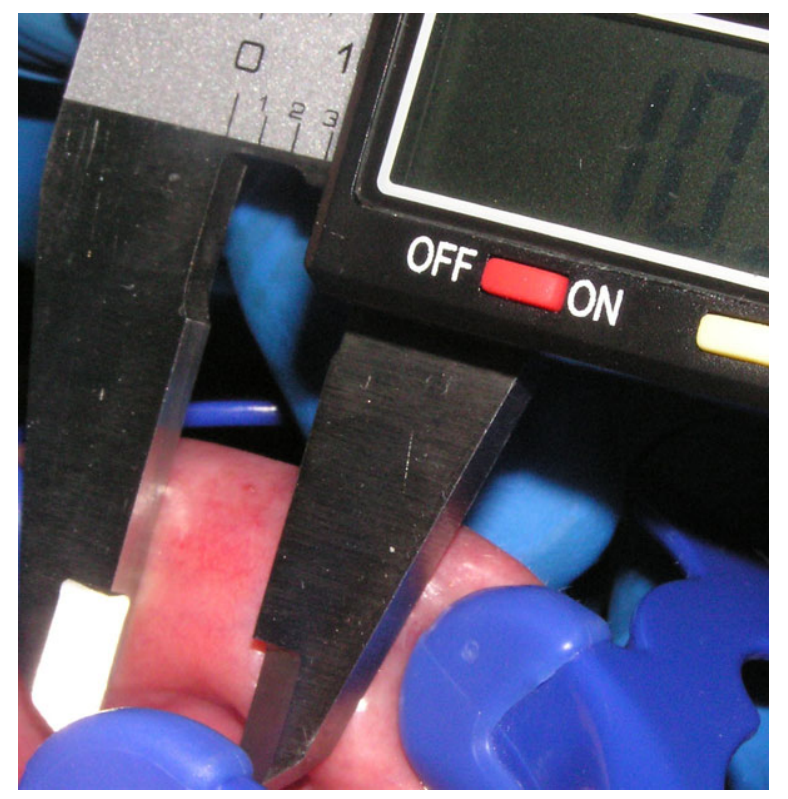

Figure 1 The largest dimension of the traumatic ulcer is measured using a digital caliper.

into contact with the polished surface. Currently, the usual care for denture-associated traumatic ulcers comprises adjustment of the margins and occlusal adaptation of the dentures, combined with instruction in home denture hygiene and careful observation.

PerioPatch $^{\circledR}$ (PeriZone ${ }^{\mathrm{TM}}$; MIS Implants Technologies, Ltd., Fair Lawn, NJ) is a novel, locally applied oral health product that acts as a barrier and provides relief from the symptoms of oral inflammation and trauma. It contains a Federal Drug Administration (FDA) approved gel that comprises several ingredients from medicinal plants and forms a protective seal over the ulcerated oral tissues from which it also absorbs wound exudates; however, to the best of our knowledge, this is the first time the effects of this patch on denture-related oral lesions have been examined.

The aims of this study were to explore the effects of PerioPatch ${ }^{\circledR}$ hydrogel wound dressing on the healing rate and pain reduction in denture-related lesions of the oral mucosa in edentulous patients, compared to usual care.

\section{Materials and methods}

\section{Ethical oversight}

The study was conducted in accordance with ethical principles, including those of the World Medical Association Declaration of Helsinki (version 2002) and additional requirements. The Ethical Board of the University of Medicine and Pharmacy "Victor Babes" Timişoara, Romania, approved the study protocol and provided oversight of the study conduct.

\section{Study population}

Participants were recruited between March and May 2012 during their routine follow-up visits after being provided with a set of complete dentures in the Department of Prosthodontics, Faculty of Dentistry, Timişoara, Romania. Fifth-year dental

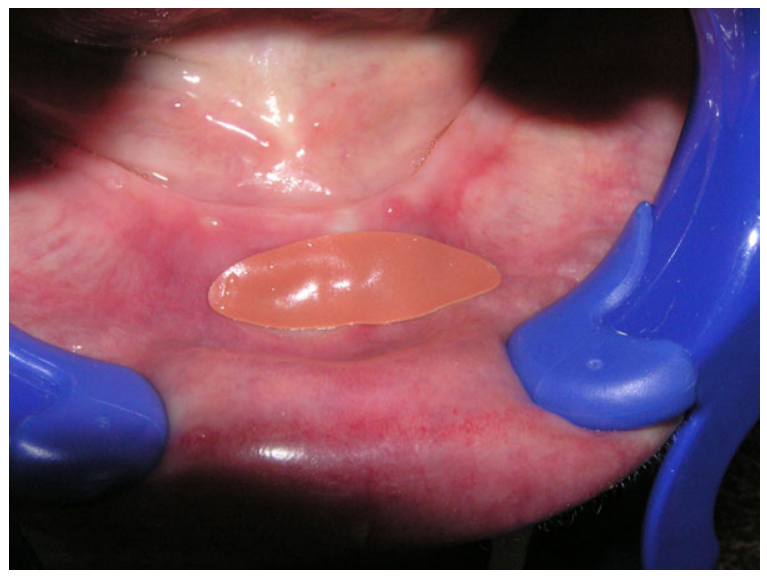

Figure 2 PerioPatch ${ }^{\circledR}$ wound healing hydrogel patch applied over the lesion.

students had completed the prosthodontic treatment under supervision by three faculty prosthodontists (AJ, LG, and RE). Eligibility for study inclusion included having at least two denture-related ulcerative lesions and no other signs or symptoms of denture stomatitis. The participants also had to be able to provide written informed consent and to follow instructions associated with the study. Persons with ulcers unrelated to their new dentures or with oral lesions related to other medical conditions were excluded.

\section{Study protocol}

The study was a clinical, nonrandomized study in which each participant served as his or her own control. In each participant, the ulcer lesion with the greatest dimension received the gel patch treatment in addition to usual care (test lesion), and the next largest lesion acted as a control lesion by receiving usual care, exclusively. If a patient had more than two lesions, the remaining lesions received usual care, but were not used as control lesions. Participants provided medical history (diabetes, other systemic diseases, and health behaviors such as cigarette smoking) and were given a brief physical examination (blood pressure, height, and weight). Denture-related lesions caused by defective or overextended margins or by a rough surface of complete new dentures were identified. The largest dimension was marked and measured by a single operator (AJ), using a digital caliper (Fig 1). Digital photographs of each lesion were also taken. The same operator adjusted denture margins and occlusion, upon which the largest lesion was covered with a hydrogel patch (test), while the smaller lesion was left to heal without any further treatment (control). Consultation with the digital photographs ensured the correct control lesion was measured at the follow-up visits. The first patch was applied in the clinic by the prosthodontist. Participants were instructed how to correctly self-apply the remaining patches. During the first 24 hours, they replaced the patch every 8 hours and wore the dentures placed over the patch. Thereafter, the patches were replaced in 1-day intervals for 3 additional days. During the night, the dentures were immersed in water after the standard cleaning procedures. Participants were instructed to not take any other medication for the ulcers. 
Table 1 Characteristics of study participants ( $N=23$ )

\begin{tabular}{|c|c|c|c|}
\hline Characteristic & All $(N=23)$ & Diabetes $(n=10)$ & No Diabetes $(n=13)$ \\
\hline \multirow[t]{2}{*}{ Age (mean $\pm S D$ ), years [range] } & $70.3( \pm 11.5)$ & $60( \pm 9.4)$ & $78.3( \pm 4.3)$ \\
\hline & [52 to 85$]$ & [52 to 78$]$ & [70 to 85$]$ \\
\hline Women (n [\%]) & $12(52.2)$ & $6(60.0)$ & $6(46.2)$ \\
\hline \multirow[t]{2}{*}{ Body mass index (BMI) (mean \pm SD), $\mathrm{kg} / \mathrm{m}^{2}$ [range] } & $26.6( \pm 3.3)$ & $29.9( \pm 1.7)$ & $24.1( \pm 1.5)$ \\
\hline & [22 to 32] & [27 to 32] & [22 to 26$]$ \\
\hline Type 2 diabetes (n [\%]) & $10(43.5)$ & $100 \%$ & $0 \%$ \\
\hline Current smoker (n [\%]) & $5(21.7)$ & $4(40.0)$ & $1(7.7)$ \\
\hline \multirow[t]{2}{*}{ Systolic blood pressure (mean \pm SD), mm Hg [range] } & $129.0( \pm 4.8)$ & $128.5( \pm 5.6)$ & $129.4( \pm 4.2)$ \\
\hline & [118 to 136$]$ & [118 to 135$]$ & [120 to 136$]$ \\
\hline \multirow[t]{2}{*}{ Diastolic blood pressure (mean $\pm \mathrm{SD}$ ), $\mathrm{mm} \mathrm{Hg}$ [range] } & $80.8( \pm 4.6)$ & $79.5( \pm 5.3)$ & $81.8( \pm 3.8)$ \\
\hline & [69 to 87] & [69 to 85] & [75 to 87] \\
\hline
\end{tabular}

$\mathrm{SD}$, standard deviation; $\mathrm{N}$, total number; $\mathrm{n}$, number in subgroup.

Table 2 Changes since baseline in the greatest dimension of lesions treated without and with hydrogel patch in all participants $(\mathrm{N}=23)$ and in those with type 2 diabetes only $(n=10)$

\begin{tabular}{|c|c|c|c|c|c|c|}
\hline & \multicolumn{3}{|c|}{ All participants ( $N=23$ ) } & \multicolumn{3}{|c|}{ Participants with diabetes $(n=10)$} \\
\hline & \multicolumn{3}{|c|}{ Baseline greatest dimension } & \multicolumn{2}{|c|}{ Baseline greatest dimension } & \\
\hline & $\begin{array}{l}\text { Lesions without patch } \\
\text { (mean } \pm \mathrm{SD}) \mathrm{mm} \\
\text { (range }[\mathrm{mm}] \text { ) }\end{array}$ & $\begin{array}{l}\text { Lesions with patch } \\
\text { (mean } \pm \mathrm{SD}) \mathrm{mm} \\
\text { (range }[\mathrm{mm}] \text { ) }\end{array}$ & & $\begin{array}{l}\text { Lesions without patch } \\
\text { (mean } \pm \mathrm{SD}) \mathrm{mm} \\
\text { (range }[\mathrm{mm}] \text { ) }\end{array}$ & $\begin{array}{l}\text { Lesions with patch } \\
\text { (mean } \pm \mathrm{SD}) \mathrm{mm} \\
\text { (range }(\mathrm{mm}])\end{array}$ & \\
\hline & $3.9 \pm 1.1(2.0$ to 7.0$)$ & $7.1 \pm 1.7(4.3$ to 10.2$)$ & & $2.0 \pm 1.0(2.0$ to 5.3$)$ & $7.9 \pm 1.6(5.2$ to 10.2$)$ & \\
\hline & \multicolumn{3}{|c|}{ Change since baseline } & \multicolumn{3}{|c|}{ Change since baseline } \\
\hline & $\begin{array}{l}\% \text { Change in } \\
\text { lesion without } \\
\text { patch (Mean } \pm \text { SD) }\end{array}$ & $\begin{array}{l}\% \text { Change in } \\
\text { lesion with } \\
\text { patch (Mean } \pm \text { SD) }\end{array}$ & $p$ & $\begin{array}{l}\% \text { Change in } \\
\text { lesion without } \\
\text { patch (Mean } \pm \text { SD) }\end{array}$ & $\begin{array}{l}\% \text { Change in } \\
\text { lesion with } \\
\text { patch (Mean } \pm \text { SD) }\end{array}$ & $p$ \\
\hline Day 1 & $10.4 \pm 10.2$ & $23.8 \pm 11.5$ & $p<0.001$ & $10.8 \pm 9.7$ & $20.1 \pm 8.6$ & $p<0.001$ \\
\hline Day 7 & $55.8 \pm 9.3$ & $73.8 \pm 10.4$ & $p<0.001$ & $55.7 \pm 9.7$ & $74.0 \pm 8.1$ & $p<0.001$ \\
\hline
\end{tabular}

$\mathrm{SD}$, standard deviation.

The greatest dimension of the lesions was again measured by digital caliper 1 and 7 days after baseline. At the follow-up visits, the pain level for each lesion was assessed using the following question: "Did your pain improve? (Yes/No)."

\section{The patch}

The PerioPatch ${ }^{\circledR}$ is an oval hydrogel patch that measures $25 \mathrm{~mm} \times 8 \mathrm{~mm}$ at its widest points (Fig 2). Applied directly on the lesion, it adheres to the mucosal surface. According to the manufacturer, the gel patch contains ethylcellulose, polyacrylic acid, Sambucus nigra (black elderberry), castor oil, acacia gum, methyl hydroxylpropyl cellulose, glycerol, strawberry flavor, Centella asiatica, titanium dioxide, Echinacea purpurea (purple coneflower), polysorbate 80, and CI 77491.

\section{Outcome measures}

The primary outcome measure was the change since baseline in the lesion's greatest dimension on days 1 and 7 (healing rate). The secondary outcome was improvement in ulcer-associated pain since last visit.

\section{Data analysis}

Since relative change is not normally distributed, the Wilcoxon signed rank test was used to compare measures from baseline to days 1 and 7. The Mann-Whitney rank sum test was used to compare participants with type 2 diabetes to those without diabetes. Significance was defined as $p<0.05$. All analyses were performed using SAS $^{\circledR} 9.2$ (SAS Institute, Inc., Cary, NC).

\section{Results}

All 23 enrolled individuals had needed replacement of old complete dentures because of ill-fitting denture bases and/or worn denture teeth. Participants were on average 70 years old, about half were women, one-fifth currently smoked cigarettes, and $40 \%$ had type 2 diabetes mellitus (Table 1). Mean systolic blood pressure was elevated, and overweight/obesity-defined as body mass index (BMI) of 25 or greater-was prevalent.

Table 2 shows the greatest lesion dimensions in the treatment and control groups. Consistent with the study design, lesions assigned to hydrogel patch application were significantly larger at baseline $(7.1 \pm 1.7 \mathrm{~mm})$ than the control lesions 
$(3.9 \pm 1.1 \mathrm{~mm})(p<0.001)$ in all participants as well as in those with diabetes only $(7.9 \pm 1.6 \mathrm{~mm}$ vs. $2.0 \pm 1.0 \mathrm{~mm})$ $(p=0.002)$. The healing rate, expressed as percent reduction in the greatest lesion dimension since baseline, was significantly higher on day 1 for lesions treated with the patch compared to usual care $(24 \%$ vs. $10 \%, p<0.001)$ (Fig 3). This difference was maintained after 7 days, as demonstrated by a $74 \%$ reduction with the patch compared to $56 \%$ in the control group $(p<0.001)$ (Table 2). There were no significant differences in the baseline dimensions of the lesions treated with the patch or only with usual care, nor in lesion size reduction, in patients with diabetes compared to patients without diabetes $(p=0.10$ and $p=0.91$, respectively).

Figure 4 illustrates the actual size of the greatest dimension of each lesion treated by standard care (without a patch) (panel A) and with a medicated hydrogel patch (panel B) at baseline, day 1 , and day 7 , respectively. The mean size of the lesions without a patch was $3.8 \pm 0.9 \mathrm{~mm}, 3.4 \pm 0.8 \mathrm{~mm}$, and $1.7 \pm 0.6 \mathrm{~mm}$ at baseline, Day 1, and Day 2, respectively, vs. $7.2 \pm 1.6 \mathrm{~mm}$, $5.5 \pm 1.4 \mathrm{~mm}$, and $1.9 \pm 0.9 \mathrm{~mm}$ for those with the patch.

Participants reported significant improvement in pain level associated with $65 \%$ of lesions treated with the hydrogel patch on Day 1 compared to only $30 \%$ of the control lesions. Pain improvement was observed in both groups on Day 7 .

\section{Discussion}

In this exploratory study, we found that local treatment by application of a hydrogel patch on denture-related oral ulcerations was associated with significantly higher rates of healing and faster decrease in pain level compared to usual care. PerioPatch ${ }^{\circledR}$ is marketed for application on periodontal tissue to provide relief from symptoms of inflammation; however, its use to promote healing of denture-related lesions and to provide accelerated pain relief for individuals with such lesions is novel. Wearing dentures improves quality of life and restores mastica- tory function in edentulous patients; however, the prevalence of oral mucosal lesions is reported to be higher in denture wearers than in nonwearers. ${ }^{1,2}$ With advancing age, the oral epithelium becomes thinner, and collagen synthesis decreases. Both contribute to reduced resilience and capacity for tissue regeneration in the oral mucosa, which consequently becomes more vulnerable to mechanical damage and demonstrates significantly lower tolerance to injury and irritation. ${ }^{5}$

In addition, coexistence of nutritional deficiencies, various systemic diseases, use of xerogenic medications, salivary quantitative and qualitative changes, tobacco use, and poor oral hygiene in the elderly may further reduce their defense against these injuries. ${ }^{4,5}$ In such circumstances, mechanical irritations result in more severe and recurrent oral lesions.

Defective or ill-fitting dentures may induce acute and chronic irritations of the oral mucosa. ${ }^{6,7}$ Denture-related ulcers are characterized by loss of tissue that affects both the epithelium and underlying connective tissue,${ }^{8}$ may vary in size and dimension, are characterized by a white or yellowish central clear area with an erythematous halo, and are generally painful. ${ }^{9-11}$ Important consequences of denture-related mucosal ulcerations include increase of the accumulation of plaque on the dentures, Candida infection, inflammatory or reactive hyperplasia, poor denture retention, mechanical trauma, ${ }^{12,13}$ poor nutrition, and general decrease in well-being. Therefore, effective treatment approaches to promote a rapid healing process of denturerelated ulcers and prevent their consequences are needed. ${ }^{12,13}$

Denture-related lesions most commonly develop within 1 to 2 days after insertion of new dentures, but can also occur in old, worn, ill-fitting dentures. As mentioned, usual care currently involves more frequent visits and regular examination of the oral cavity in complete denture wearers, occlusal adjustment, and denture margin adjustments.

The hydrogel patch may promote the healing of ulcers through several possible mechanisms. The PerioPatch ${ }^{\circledR}$ contains substances from several medicinal plants and other

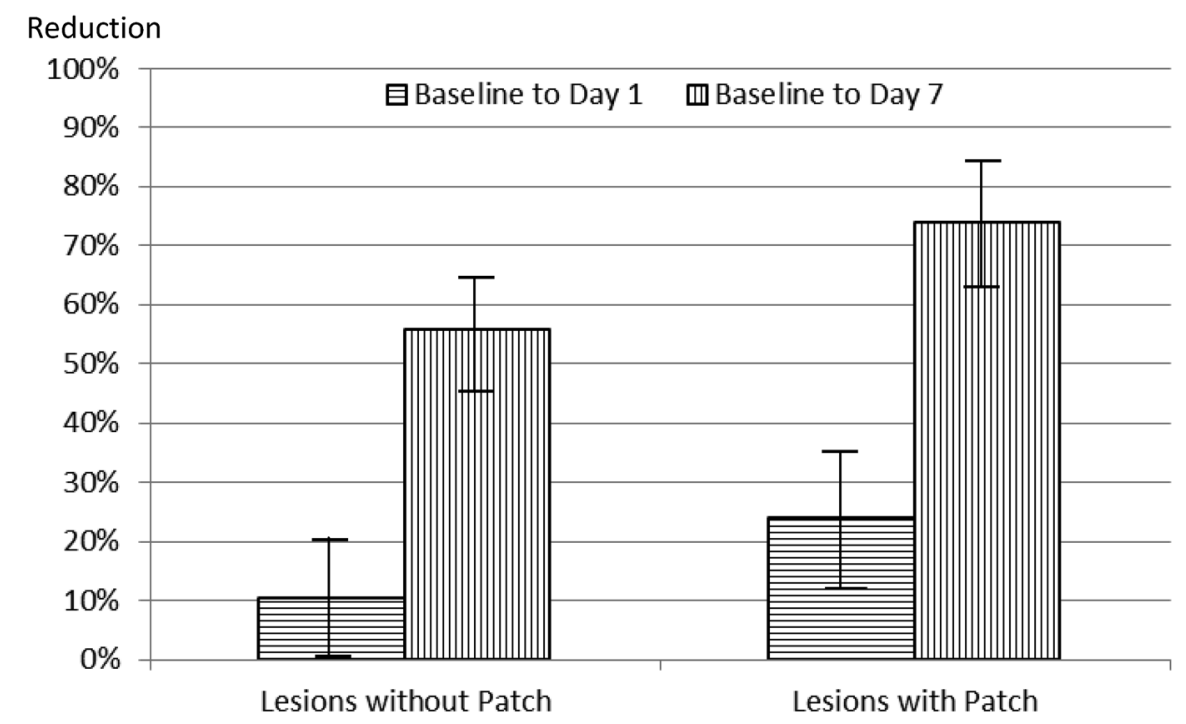

Figure 3 Mean decrease in greatest dimension of lesions treated without patch (control) and with patch (test) over time. 

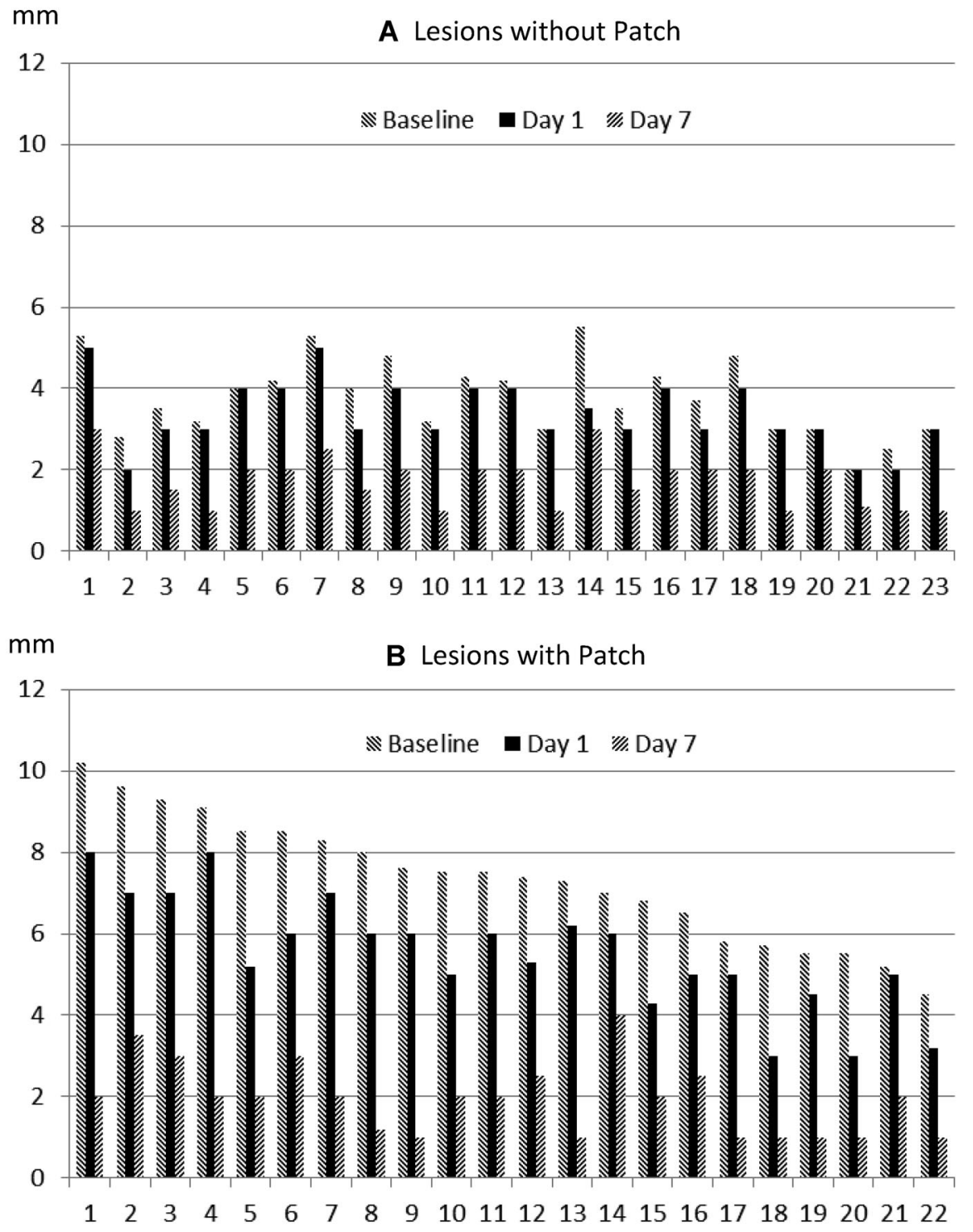

Figure 4 Changes since baseline in the greatest dimension of lesions treated without patch (A) and with patch (B).

ingredients suggested to enhance healing by absorbing wound exudates and by reducing tissue inflammation. In addition, the patch forms a protective seal over the irritated and inflamed oral tissues, providing protection from further irritation caused by normal activities such as speaking and eating. It is therefore possible that part of the observed improved healing associated with the hydrogel patch may have been due to the "bandage" effect of the patch and not necessarily attributable to its medicinal components. Such bandage would also protect against additional pain caused by mechanical irritation and possibly against infections; however, the differences in the rates of both healing and pain reduction caused by the application of the patch are so great that this finding supports an active role of the patch.

Various hydrogels are used in medicine for wound dressing and transdermal drug delivery. Similar to our results, a
1996 study reported that patients claimed reduced healing time of aphthous ulcers due to treatment with bioadhesive hydrogel patches made from a pharmaceutical grade cellulose derivative. ${ }^{14}$ In contrast, application of topical gel with and without triester glycerol oxide (without containment in a patch) in denture-related mucosal injuries was not effective for treating injuries from new full dentures. ${ }^{15}$ No prior reports have described the use of any hydrogel patch for denture-related ulcerations.

Recent studies report that diabetes mellitus is a risk factor for development of ulcerations related to complete dentures. ${ }^{16}$ However, other studies found no differences in healing rates in oral lesions in people with diabetes and those free of diabetes. ${ }^{4}$ Consistent with the latter observation, this study did not identify any significant difference in lesion size or in their rate of healing 
between patients with diabetes compared with those with no diabetes, with either the patch or usual care; however, data from this small sample size may not have possessed the statistical power to detect any actual difference.

A clear benefit of the hydrogel patch was observed in both the rate of the healing of the traumatic ulcers and in the associated pain, compared to the lesions receiving usual care. Several factors may affect healing rates, including smoking and presence of diabetes, especially poorly controlled diabetes; however, given the fact that in this study each patient acted as his/her own control, it is unlikely that such factors could have affected the results.

Limitations include the small sample size, which prevented more comprehensive subgroup analyses and statistical modeling to identify additional contributing factors. In addition, this was a nonrandomized clinical study in which we used the lesion with the greatest dimension as the treatment lesion and compared that to the next largest lesion as the control lesion in the same patient. Although assigning the larger lesion as the test lesion may have affected the results and could have introduced some bias, this design was used for ethical reasons to increase the likelihood for healing and pain relief for these patients. The level of glycemic control in participants with diabetes was not available for inclusion in our analyses. Hence, it is possible that people with uncontrolled or poorly controlled diabetes actually might have experienced slower healing than those without diabetes or with well-controlled diabetes that this study was not able to detect.

Future studies are needed to confirm these preliminary findings. Such studies should be conducted among many more individuals, enabling subgroup analyses, for instance between groups with different levels of glycemic control and other potential confounders, such as tobacco-smoking habits. Furthermore, future studies assessing the efficacy of an unmedicated patch as a control could explore whether the medication containing PerioPatch ${ }^{\circledR}$ provides better relief than an unmedicated bandage whose effect would exclusively consist of mechanical protection of the ulcer. Larger studies should also investigate whether the performance of medicated and unmedicated patches are indeed superior to the current standard care that does not involve the use of any patch.

\section{Conclusion}

This report presents the novel findings that the addition of locally applied hydrogel patches (PerioPatch ${ }^{\circledR}$ ) to usual care significantly promotes the rate of healing of denture-related mucosal ulcers, as well as accelerates accompanying pain reduction. These effects were present at both 1 and 7 days after the initial application, compared to exclusively usual care that currently consists of adjustment of the dentures' margins and occlusion and polishing any rough spots, supported by instruction in home denture hygiene and professional monitoring. There were no differences in healing rates between participants with and without diabetes at either 1 or 7 days upon treatment initiation. These results provide evidence to guide further research with larger sample sizes, which may support initiation of routine use of hydrogel patch treatment in patients with denturerelated ulcers. In turn, such improvement of future standard of care could result in more rapid decrease in suffering and lead to faster improvement in quality of life for individuals with denture-related ulcers.

\section{Acknowledgments}

Whereas no financial support was received for this study, the authors are grateful for the donation of all the PerioPatch ${ }^{\circledR}$ PeriZone ${ }^{\mathrm{TM}}$ hydrogel patches from MIS Implants Technologies, Ltd. We also wish to acknowledge the fifth-year dental students during the 2011 to 2012 academic year at the Faculty of Dentistry, University of Medicine and Pharmacy "Victor Babes" Timisoara, Romania, their instructors from the Department of Prosthodontics, and the participating patients who made this study possible.

\section{References}

1. Tuominen R: Oral health in relation to wearing removable dentures provided by dentists, denturists and laboratory technicians. J Oral Rehabil 2003;30:743-748

2. Freitas JB, Gomez RS, De Abreu MH, et al: Relationship between the use of full dentures and mucosal alterations among elderly Brazilians. J Oral Rehabil 2008;35:370-374

3. Saranda DL: Sequelae of ill fitting dentures. In Sarandha DL (ed): Textbook of Complete Denture Prosthodontics (ed 1). New Delhi, Jaypee Brothers Medical Publishers, 2007, pp. 135-137

4. Cristina de Lima D, Nakata GC, Balducci I, et al: Oral manifestations of diabetes mellitus in complete denture wearers. J Prosthet Dent 2008;99:60-65

5. Jainkittivong A, Aneksuk V, Langlais RP: Oral mucosal conditions in elderly dental patients. Oral Dis 2002;8:218-223

6. Coelho CM, Sousa YT, Dare AM: Denture-related oral mucosal lesions in a Brazilian school of dentistry. J Oral Rehabil 2004;31:135-139

7. Kivovics P, Jahn M, Borbely J, et al: Frequency and location of traumatic ulcerations following placement of complete dentures. Int J Prosthodont 2007;20:397-401

8. Jainkittivong A, Aneksuk V, Langlais RP: Oral mucosal lesions in denture wearers. Gerodontology 2010;27:26-32

9. Bascones A, Figuero E, Esparza G: Ulceras orales. Med Clin (Barc) 2005;125:590-597

10. Cawson RA, Odell EW: Diseases of the oral mucosa: Non-infective stomatitis. In Cawson RA (ed): Cawson's Essentials of Oral Pathology and Oral Medicine (ed 8). Edinburgh, Churchill Livingstone, 2008, pp. 220

11. Talacko AA, Gordon AK, Aldred MJ: The patient with recurrent oral ulceration. Aust Dent J 2010;55 (Suppl 1):14-22

12. Coelho CM, Zucoloto S, Lopes RA: Denture-induced fibrous inflammatory hyperplasia: a retrospective study in a school of dentistry. Int J Prosthodont 2000;13:148-151

13. Correa L, Frigerio ML, Sousa SC, et al: Oral lesions in elderly population: a biopsy survey using 2250 histopathological records. Gerodontology 2006;23:48-54

14. Mahdi AB, Coulter WA, Woolfson AD, et al: Efficacy of bioadhesive patches in the treatment of recurrent aphthous stomatitis. J Oral Pathol Med 1996;25:416-419

15. Geckili O, Bektas-Kayhan K, Eren P, et al: The efficacy of a topical gel with triester glycerol oxide in denture-related mucosal injuries. Gerodontology 2012;29:e715-e720

16. Dundar N, Ilhan Kal B: Oral mucosal conditions and risk factors among elderly in a Turkish school of dentistry. Gerontology 2007;53:165-172 